This item was submitted to Loughborough's Research Repository by the author.

Items in Figshare are protected by copyright, with all rights reserved, unless otherwise indicated.

Spatial dispersive shock waves generated in supersonic flow of BoseEinstein condensate past slender body

PLEASE CITE THE PUBLISHED VERSION

LICENCE

CC BY-NC-ND 4.0

REPOSITORY RECORD

El, G.A., and A.M. Kamchatnov. 2019. "Spatial Dispersive Shock Waves Generated in Supersonic Flow of Bose-einstein Condensate Past Slender Body”. figshare. https://hdl.handle.net/2134/389. 


\title{
Spatial dispersive shock waves generated in supersonic flow of Bose-Einstein condensate past slender body
}

\author{
G.A. $\mathrm{El}^{1 *}$ and A.M. Kamchatnov ${ }^{2 \dagger}$ \\ 1 Department of Mathematical Sciences, Loughborough University, Loughborough LE11 $3 T$, UK \\ ${ }^{2}$ Institute of Spectroscopy, Russian Academy of Sciences, Troitsk, Moscow Region, 142190, Russia
}

(Dated: April 20, 2005)

\begin{abstract}
Supersonic flow of Bose-Einstein condensate past macroscopic obstacles is studied theoretically. It is shown that in the case of large obstacles the Cherenkov cone transforms into a stationary spatial shock wave which consists of a number of spatial dark solitons. Analytical theory is developed for the case of obstacles having a form of a slender body. This theory explains qualitatively the properties of such shocks observed in recent experiments on nonlinear dynamics of condensates of dilute alkali gases.
\end{abstract}

PACS numbers: 03.75.Lm, 32.80.Pj, 43.35.+d, 67.40.Vs 67.90.+z

In usual compressible fluid dynamics, there are two typical situations when shock waves can be generated. The first one is connected with breaking of a nonlinear wave and the second with a supersonic flow past a body (see, e.g., $[1,2]$ ). In a viscous fluid, the shock wave can be represented as a narrow region within which strong dissipation processes take place and the thermodynamic and hydrodynamic parameters of the flow undergo sharp change. If viscosity is negligibly small compared with dispersion effects, the shock discontinuity is resolved into an expanding region filled with nonlinear oscillations. A remarkable feature of such a "dispersive shock" is generation of solitons at one of its boundaries so that the whole structure can often be asymptotically represented as a "soliton train". The theory of dispersive shocks based on the Whitham nonlinear modulation theory was developed for media described by the Korteweg-de Vries equation as for the wave breaking case [3], so for the flow past a slender body [4]. In the latter case the "soliton train" represents a "fan" of oblique spatial solitons spreading downstream from the pointed part of the body.

After experimental discovery of the Bose-Einstein condensate (BEC) [5-7], it was found that its dynamics is described very well by the Gross-Pitaevskii (GP) equation (see, e.g., [8])

$$
i \hbar \frac{\partial \psi}{\partial t}=-\frac{\hbar^{2}}{2 m} \Delta \psi+U(\mathbf{r}) \psi+g|\psi|^{2} \psi,
$$

where $\psi(\mathbf{r})$ is the order parameter ("condensate wave function"), $U(\mathbf{r})$ is the potential which confines atoms of Bose gas in a trap, and $g$ is an effective coupling constant arising due to inter-atomic collisions with the $s$ wave scattering length $a_{s}, g=4 \pi \hbar^{2} a_{s} / m, m$ being the atomic mass. Obviously, the GP equation (1) combines the dispersive and nonlinear effects, and the corresponding properties of BEC dynamics have been investigated in a number of papers (see for review, e.g., [8]). In particular, if $g>0$, then existence of dispersive shocks can be expected, their theory was developed in $[9,10]$ and they were observed in a recent experiment [11]. Although in the experiment [11] the shock flow was strongly disturbed by vortices arising due to rotation of the condensate, we were informed [12] about unpublished results of experiments on shocks in non-rotating BEC, and these results agree qualitatively with the theory [9].

Besides the experiments on generation of shocks after wave breaking of BEC's flow, in [12] the experiments were performed on BEC's flow past an obstacle after release of BEC from the confining potential. The problem of superflow past a body has been studied intensely in connection with a problem of critical velocity $v_{c}$ above which superfluidity disappears (see, e.g. [13]). It was found that superfluidity is lost due to generation of vortex rings behind an obstacle which gives the estimate of critical velocity $v_{c} \sim(\hbar / d m) \ln (d / \xi)$, where $\xi=\hbar / \sqrt{2 m n_{0} g}$ is the healing length (i.e. "vortex core size"),$d$ is the size of obstacle in transverse direction, and $n_{0}$ is the density of atoms in the condensate far from the obstacle. For large obstacles with $d \gg \xi$ this estimate gives the critical velocity much less than the sound velocity $c_{s}=\hbar / \sqrt{2} m \xi$. This transition to dissipation in quantum fluids described by the GP equation (1) was confirmed by numerical study [14] where it was found that indeed vortices are generated at velocities above the critical one about $\sim 0.45 c_{s}$ for $d=10 \xi$. Since the radius of vortex rings (or distance between vortices in pairs in two dimensions) is about the obstacle size $d$, this mechanism of vortices emission becomes ineffective for $d \sim \xi$, and for such small bodies ("impurities") the critical velocity arises due to Cherenkov emission of sound waves and coincided, hence, with the sound velocity $c_{s}$ [15]. Obviously, emission of waves in a supersonic flow past an obstacle remains effective also for large obstacles with $d \gg \xi$, but in this case emitted waves are not linear sound waves and, instead of a Cherenkov cone, we arrive at above mentioned dispersive shock consisting of oblique spatial solitons. Actually, these shock waves have been observed in the experiment [12] where the wave pattern consists of a series of distinct oblique traces which cannot be attributed to a linear Cherenkov radiation. An easy estimate shows that, after long enough time of expansion 
the velocity of the flow past a body is much greater than the local sound velocity in BEC near this body. Indeed, the flow velocity $u$ in a free expansion has the order of magnitude of the sound velocity at the center of BEC before its release and it is known that the sound speed in BEC is proportional to the square root of density. Since for the expansion time $t \gg \omega_{\perp}^{-1}\left(\omega_{\perp}\right.$ is the radial trap frequency before expansion) the density is proportional to $t^{-2}$, we find that the ratio of the expansion flow velocity to the local sound speed, i.e. the Mach number $M$, is about

$$
M \sim \omega_{\perp} t \gg 1
$$

for $t \gg \omega_{\perp}^{-1}$. Thus, the flow past a body in the experiment [12] is supersonic and, correspondingly, formation of a "fan" of spatial solitons is expected. Motivated by the results of this experiment and above physical argumentation, we shall develop here the theory of spatial dispersive shocks on the basis of the GP equation (1).

In accordance with the experiment $[11,12]$, we consider a two-dimensional flow of the condensate, so that the condensate wave function $\psi$ depends on only two spatial coordinates $\mathbf{r}=(x, y)$. To simplify the theory, we assume that the characteristic size of the body is much less than its distance from the center of the trap, so that incoming flow can be considered as uniform with constant density $n_{0}$ of atoms and constant velocity $\mathbf{u}_{0}$ directed parallel to $x$ axis. It is convenient to transform Eq. (1) to a "hydrodynamic" form by means of the substitution

$$
\psi(\mathbf{r}, t)=\sqrt{n(\mathbf{r}, t)} \exp \left(\frac{i}{\hbar} \int^{\mathbf{r}} \mathbf{u}\left(\mathbf{r}^{\prime}, t\right) d \mathbf{r}^{\prime}\right),
$$

where $n(\mathbf{r}, t)$ is density of atoms in BEC and $\mathbf{u}(\mathbf{r}, t)$ denotes its velocity field, and to introduce dimensionless variables $\tilde{x}=x / \sqrt{2} \xi, \tilde{y}=y / \sqrt{2} \xi, \tilde{t}=\left(c_{s} / 2 \sqrt{2} \xi\right) t, \tilde{n}=$ $n / n_{0}, \tilde{\mathbf{u}}=\mathbf{u} / c_{s}$, where numerical constants are introduced for future convenience. As a result of this transformation we obtain the system (we omit tildes for convenience of the notation)

$$
\begin{array}{r}
\frac{1}{2} n_{t}+\nabla(n \mathbf{u})=0, \\
\frac{1}{2} \mathbf{u}_{t}+(\mathbf{u} \nabla) \mathbf{u}+\nabla n+\nabla\left[\frac{(\nabla n)^{2}}{8 n^{2}}-\frac{\Delta n}{4 n}\right]=0
\end{array}
$$

(where $\nabla=\left(\partial_{x}, \partial_{y}\right)$ ) which should be solved with the boundary conditions

$$
n=1, \quad \mathbf{u}=(M, 0) \quad \text { at } \quad x \rightarrow-\infty
$$

for incoming flow and

$$
\left.\mathbf{u} \cdot \mathbf{N}\right|_{S}=0
$$

at the body surface $S$, where $\mathbf{N}$ denotes a unit vector of outer normal to the surface $S$. Under our assumption that the size of the body is much less than the distance from the center of the cylindrically symmetrical flow, the characteristics of the flow near the body change with the time very slowly, so that the arising wave pattern can be considered as a quasi-stationary one. Hence, we can confine ourselves to the stationary solutions of our problem (4)-(6) and replace Eqs. (4) by their stationary versions for two-dimensional stationary velocity field $\mathbf{u}=(u(x, y), v(x, y))$ :

$$
\begin{array}{r}
(n u)_{x}+(n v)_{y}=0 \\
u u_{x}+v u_{y}+n_{x}+\left(\frac{n_{x}^{2}+n_{y}^{2}}{8 n^{2}}-\frac{n_{x x}+n_{y y}}{4 n}\right)_{x}=0 \\
u v_{x}+v v_{y}+n_{y}+\left(\frac{n_{x}^{2}+n_{y}^{2}}{8 n^{2}}-\frac{n_{x x}+n_{y y}}{4 n}\right)_{y}=0 .
\end{array}
$$

If the body is symmetric with respect to $x$ axis and the form of its boundary is given by $y= \pm F(x), F(0)=$ $0, F(l)=0, l$ being the longitudinal size of the body, then we can confine ourselves to consideration of only the upper half-plane $y>0$, so that $\mathbf{N} \propto\left(F^{\prime}(x),-1\right)$, and the boundary conditions (5), (6) are transformed to

$$
\begin{gathered}
n=1, \quad u=M, \quad v=0 \quad \text { at } \quad x \rightarrow-\infty, \\
v=u F^{\prime}(x) \quad \text { at } \quad y=F(x) .
\end{gathered}
$$

The system (7)-(9) is still too complicated for analytical treatment. However, the flow is supposed to be supersonic (see (2)) which allows us to asymptotically transform Eqs. (7)-(9) to a much simpler problem of 1D "unsteady" flow along $y$ axis with the scaled $x$ coordinate playing the role of "time" [16]. To this end, we substitute into Eqs. (7) new variables

$$
u=M+u_{1}+O(1 / M), \quad T=x / 2 M, \quad Y=y,
$$

assuming $M^{-1} \ll 1$. Then to leading order we obtain

$$
\begin{gathered}
\frac{1}{2} n_{T}+(n v)_{Y}=0 \\
\frac{1}{2} v_{T}+v v_{Y}+n_{Y}+\left(\frac{n_{Y}^{2}}{8 n^{2}}-\frac{n_{Y Y}}{4 n}\right)_{Y}=0 \\
\frac{1}{2} u_{1 T}+v u_{1 Y}=0 .
\end{gathered}
$$

Equations (11) represent the hydrodynamic form of 1D nonlinear Schrödinger (NLS) equation $i \Psi_{T}+\Psi_{Y Y}-$ $2|\Psi|^{2} \Psi=0$ for $\Psi=\sqrt{n} \exp \left(i \int^{Y} v\left(Y^{\prime}, t\right) d Y^{\prime}\right)$, and we can apply well-known methods to their study. If $n$ and $v$ are found, then the correction $u_{1}$ to the $x$ component of velocity can be calculated with the use of Eq. (12).

It is remarkable that in the case of a slender body, for which $M \alpha \lesssim 1$ where $\alpha=\max \left|F^{\prime}(x)\right|$, the boundary condition (9) reduces (to leading order in $M^{-1}$ ) to

$$
v=v_{p}=\frac{1}{2} d f / d T \quad \text { at } \quad Y=f(T),
$$


where $f(T)=F(2 \sqrt{2} \xi M T) / \sqrt{2} \xi$, and it does not contain the $u$-variable in this approximation.

Thus, we have reduced the problem of flow past a slender body to the classical "piston" problem for 1D flow along a tube with a piston moving according to the law (13). In contrast to the classical gas dynamics, the piston problem is now posed for a dispersive equations(11) and its full analytic treatment is technically quite involved (see the analogous theory for the $\mathrm{KdV}$ equation case in [4]). However, in the region far enough from the body where the shock develops into a "fan" of spatial solitons well separated from each other one can take advantage of a more simple asymptotic method of Ref. [17]. The

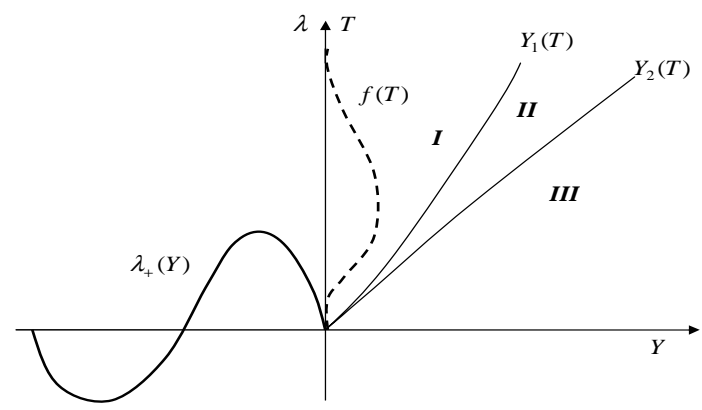

FIG. 1: $(Y, T)$-plane of the piston problem $(Y>0)$ and the equivalent initial data $\lambda_{+}(Y)(Y<0)$. Dashed line: the "piston" trajectory $Y=f(T)$.

$(Y, T)$-plane of the auxiliary piston problem can be subdivided into three distinct regions, see Fig. 1. Generally, for $Y>f(T)$, the "gas" is put into motion by the "piston" moving according to Eq. (13) and in the region $I$ near the "piston" the gas motion can be described by the dispersionless limit

$$
\frac{1}{2} n_{T}+(n v)_{Y}=0, \quad \frac{1}{2} v_{T}+v v_{T}+n_{Y}=0
$$

of Eqs. (11). But formal solution of the dispersionless equations cannot be extended to the whole $(Y, T)$-plane because the $Y$-derivatives blow up along some line in this plane. Hence, here we have to take into account the dispersion effects which lead to formation of the region II filled with nonlinear oscillations - a dispersive shock. Close to its boundary $Y=Y_{1}(T)$ facing the "piston" the oscillations tend to a train of dark solitons of the NLS equation equivalent to the system (11), and at the opposite boundary $Y=Y_{2}(T)$ the amplitude of oscillations tends to zero which corresponds to a linear "sound" wave propagating into the undisturbed region $I I I$ with $Y>Y_{2}(T)$. For $T \gg l(2 \sqrt{2} \xi M)^{-1}$ the whole structure can be asymptotically represented as a soliton train [4]. Thus, in the case of a macroscopic obstacle with characteristic size much greater than the healing length $\xi$ the Cherenkov cone "unfolds" into a "fan" of solitons.

Motion of the gas can be described most conveniently in terms of Riemann invariants (see, e.g., [17]). In the re- gions $I$ and $I I I$ of the smooth flow they are the Riemann invariants of the dispersionless system (14)

$$
\lambda_{ \pm}=\frac{1}{2} v \pm \sqrt{n},
$$

and in the dispersive shock region $I I$ they are four Riemann invariants $\lambda_{i}, i=1,2,3,4$, which parameterize the modulated periodic solution of the full system (11), and obey the corresponding Whitham modulation equations (see, e.g., [17]). The Riemann invariants (15) are constant along the corresponding families of characteristics of the system (14) and they must satisfy the matching conditions along the lines $Y=Y_{1,2}(T)$. In the undisturbed region $I I I$, where $v=0, n=1$, both Riemann invariants are constant: $\lambda_{ \pm}= \pm 1$. According to wellknown argumentation (see [1], Section 104), one of the Riemann invariants $\lambda_{i}, i=1,2,3,4$, which matches with, say, $\lambda_{-}$must be also constant in the whole region $I I$ : $\lambda_{4}=\lambda_{-}=-1$ in $I I$. On the other hand, the gas motion in the region $I$ produced by the "piston" is described by a simple wave solution of Eqs. (14) (see [1], Section 101) again with one Riemann invariant constant everywhere in $I$. It must match with $\lambda_{4}$ along the characteristic line $Y=Y_{1}(T)$ so that $\lambda_{-}=\lambda_{4}=-1$ in the whole $(Y, T)$ plane including the trajectory of the "piston". Hence, we have at the "piston" $v_{p} / 2-\sqrt{n_{p}}=-1$ which yields the gas density, $n_{p}=\left(v_{p}+2\right)^{2} / 4$, and, consequently, the values of both Riemann invariants:

$$
\lambda_{-}^{p}=-1, \quad \lambda_{+}^{p}=\frac{1}{2} d f / d T+1 \quad \text { at } \quad Y=f(T) .
$$

To use the method of Ref. [17], we have to transform these boundary conditions at the "piston" to the equivalent initial conditions at $T=0, Y<0$ (see Fig. 1). This problem for the system (14) can be easily solved using characteristics. Indeed, we have $\lambda_{-}=-1$, hence $\lambda_{+}$obeys the equation (see, e.g., [17]) $\partial \lambda_{+} / \partial T+\left(3 \lambda_{+}-1\right) \partial \lambda_{+} / \partial Y=0$ whose solution is well known $Y=\left(3 \lambda_{+}-1\right) T+\bar{Y}\left(\lambda_{+}\right)$ where a function $\bar{Y}\left(\lambda_{+}\right)$must be chosen so that the condition (16) is satisfied. This gives at once $\bar{Y}\left(\lambda_{+}\right)=$ $-\left(3 \lambda_{+}-1\right) \tau$, where $\tau$ is determined implicitly by the equation $\lambda_{+}=\frac{1}{2} f^{\prime}(\tau)+1$. Thus, we arrive at a parametric form of the initial distribution of the Riemann invariant $\lambda_{+}$:

$$
\lambda_{+}=\frac{1}{2} f^{\prime}(\tau)+1, \quad Y=f(\tau)-\left(\frac{3}{2} f^{\prime}(\tau)+2\right) \tau .
$$

Now we can use the asymptotic method of Ref. [17] according to which the "initial pulse" evolves eventually into the soliton train where each soliton is parameterized by the eigenvalue $\lambda_{k}$ of the generalized Bohr-Sommerfeld quantization rule

$$
\begin{aligned}
\oint \sqrt{\left(\lambda_{k}-\lambda_{+}\right)\left(\lambda_{k}-\lambda_{-}\right)} d Y & =2 \pi\left(k+\frac{1}{2}\right), \\
k & =0,1, \ldots, K,
\end{aligned}
$$

where in our case $\lambda_{+}(Y)$ is given by Eq. (17), $\lambda_{-}=-1$, and the integration is taken over the cycle around two 
turning points where the integrand functions vanishes. Returning to the spatial coordinates (10), we find the profile of the $\lambda_{k}$-soliton in the train as (see [17])

$$
n_{k}(x, y)=1-\frac{1-\lambda_{k}^{2}}{\cosh ^{2}\left[\sqrt{1-\lambda_{k}^{2}}\left(y-\left(\lambda_{k} / M\right) x\right)\right]},
$$

that is the "fan" of spatial dark solitons in the shock is made of soliton "feathers" lying along the lines

$$
y=\left(\lambda_{k} / M\right) x, \quad k=0,1, \ldots, K,
$$

in the upper half-plane and symmetric "fan" of solitons is generated in the lower half-plane.

Let us illustrate this theory by an example of a body with a parabolic profile

$$
y=F(x)=\alpha x(L-x) / M^{2}, \quad 0 \leq x \leq L,
$$

so that the initial condition (17) takes the form

$$
\lambda_{+}=\alpha\left(T_{0}-2 \tau\right)+1, \quad Y=\tau\left(2 \alpha \tau-\alpha T_{0} / 2-2\right),
$$

for $0 \leq \tau \leq T_{0}=L / 2 M$, that is in the interval $-T_{0}(2-$ $\left.3 \alpha T_{0} / 2\right) \leq Y \leq 0$, and $\lambda_{+}=1$ outside this interval. Its part with $\lambda_{+}>1$ evolves into non-solitonic wave which does not give any contribution into the shock. However, its "well" part $\lambda_{+}<1$ leads to the bound states in the spectral problem (18) and, hence, to the train of spatial solitons (19) generated in the shock. Integral in (18) is calculated in closed form which gives the equation

$$
\frac{2 \sqrt{2}}{5 \pi \alpha}\left(1+\frac{2}{3} \lambda_{k}-\frac{27}{12} \alpha T_{0}\right)\left(\alpha T_{0}-1+\lambda_{k}\right)^{3 / 2}=k+\frac{1}{2},
$$

and its roots $\lambda_{k}$ must lie in the interval $1-\alpha T_{0}<\lambda_{k}<1$. The greatest root $\lambda_{K}$ has a value close to unity so that the number of solitons in the shock is given approximately by (23) with $\lambda_{k}=1, k=K$. To transform this estimate to dimensional variables, we take $l=\sqrt{2} \xi L=1 \sqrt{2} \xi M T_{0}$ as a longitudinal size of the obstacle, $d=\sqrt{2} \xi f\left(T_{0} / 2\right)=$ $\xi \alpha T_{0}^{2} / \sqrt{2}$ as its transverse size and obtain

$$
K \cong \frac{2}{5 \pi}\left(1-\frac{27}{5} \frac{M d}{l}\right) \sqrt{\frac{l d}{M \xi^{2}}} .
$$

Although this formula is derived for a slender body, we can use it as a rough estimate of a number of solitons in the shock generated in the supersonic flow past an obstacle: $K \cong$ const $\cdot\left(l d / M \xi^{2}\right)^{1 / 2}$, where $K$ must be much greater than unity. The most shallow dark soliton lies near the Cherenkov cone with angle $\theta \cong 1 / M=c_{s} / u_{0}$ with respect to the direction of the flow. The resulting pattern is shown in Fig. 2.

In conclusion, we have developed the theory of spatial dispersive shock waves generated by a flow of BoseEinstein condensate past a slender body. The theory

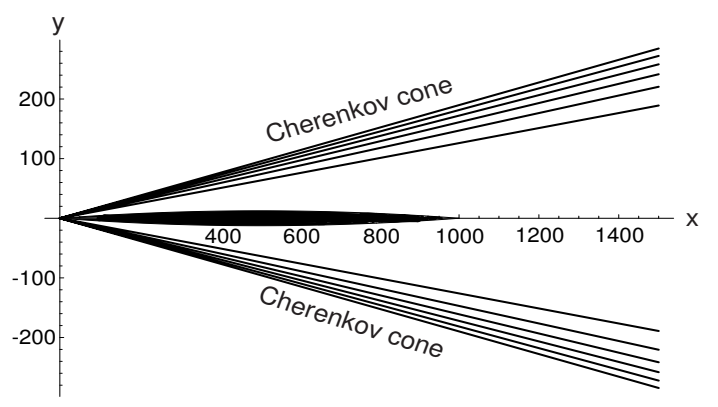

FIG. 2: The pattern of dispersive shocks generated by a supersonic flow past a slender body (black cigar-shaped figure). The oblique lines represent the traces of dark solitons in the $(x, y)$-plane.

predicts formation of a series of oblique spatial solitons in the flow and explains qualitatively the shock patterns observed in the experiment [12].

We are grateful to E.A. Cornell and P. Engels for information about the results of the experiments of JILA group prior to publication. AMK thanks RFBR (grant 05-02-17351) for financial support.

* Electronic address: G.El@lboro.ac.uk

$\dagger$ Electronic address: kamch@isan.troitsk.ru

[1] L.D. Landau and E.M. Lifshitz, Fluid Mechanics (Pergamon Press, Oxford, 1987).

[2] G.B. Whitham, Linear and Nonlinear Waves (WileyInterscience, New York, 1974).

[3] A.V. Gurevich and L.P. Pitaevskii, Sov. Phys. JETP, 38, 291 (1974).

[4] A.V. Gurevich et al, JETP, 81, 87 (1995); 82, 709 (1996).

[5] M.N. Anderson et al, Science, 269, 198 (1995).

[6] K.B. Davis et al, Phys. Rev. Lett. 75, 3969 (1995).

[7] C.C. Bradley, A. Sackett, and R.G. Hulet, Phys. Rev. Lett. 75, 1687 (1995); ibid 78, 985 (1997).

[8] L.P. Pitaevskii and S. Stringari, Bose-Einstein Condensates in Gases (CUP, Cambridge 2003).

[9] A.M. Kamchatnov, A. Gammal, and R.A. Kraenkel, Phys. Rev. A 69, 063605 (2004).

[10] B. Damski, Phys. Rev. A 69, 043610 (2004).

[11] T.P. Simula et al, Phys. Rev. Lett. 94, 080404 (2005).

[12] E.A. Cornell and P. Engels (private communication).

[13] P. Nozières and D. Pines, The Theory of Quantum Liquids II (Addison-Wesley, Redwood City, 1990)

[14] T. Frisch, Y. Pomeau, and S. Rica, Phys. Rev. Lett. 69, 1644 (1992).

[15] G.E. Astrakharchik and L.P. Pitaevskii, Phys. Rev. A bf 70, 013608 (2004).

[16] G.A. El, V.V. Khodorovskii, and A.V. Tyurina, Phys. Lett. A 333, 334 (2004).

[17] A.M. Kamchatnov, R.A. Kraenkel, and B.A. Umarov, Phys. Rev. E 66, 036609 (2002). 